\title{
Association between Family History of Cancer and Lung Cancer Risk among Japanese Men and Women
}

\author{
Kaoru Yoshida, ${ }^{1,2,3,4}$ Yoko Takizawa, ${ }^{1}$ Yoshikazu Nishino,${ }^{4,5}$ Satomi Takahashi, ${ }^{6}$ \\ Seiki Kanemura, ${ }^{4}$ Junko Omori, ${ }^{2}$ Hajime Kurosawa, ${ }^{3}$ Makoto Maemondo ${ }^{7,8}$ and \\ Yuko Minami ${ }^{1,4,9}$

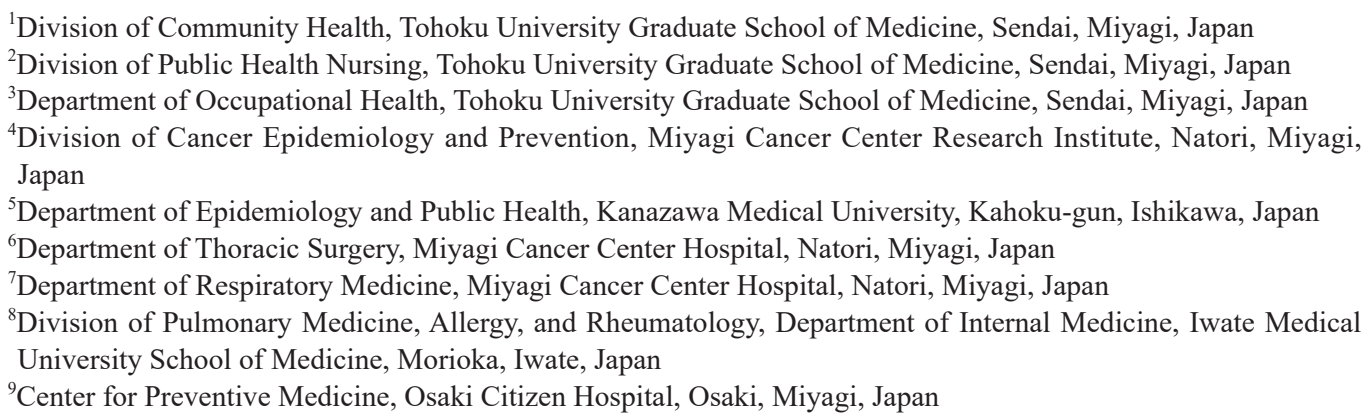

Although cigarette smoking is a major risk factor for lung cancer, genetic susceptibility may also affect lung cancer risk. To explore the role of genetic risk, this case-control study investigated the association between family history of cancer at several sites and lung cancer risk. A total of 1,733 lung cancer cases and 6,643 controls were selected from patients aged 30 years and over admitted to a single hospital in Japan between 1997 and 2009. Information on family history of cancer was collected using a self-administered questionnaire and odds ratios (ORs) were estimated by unconditional logistic regression. Family history of lung cancer in first-degree relatives was associated with an increased risk of lung cancer among both sexes. According to histology and type of relatives, a parental history of lung cancer was significantly associated with an increased risk of female adenocarcinoma $(O R=1.72)$. Stratification by smoking status revealed that this significant positive association in women was limited to ever-smokers $(\mathrm{OR}=4.13)$. In men, a history of lung cancer in siblings was significantly associated with an increased risk of small cell carcinoma $(O R=2.28)$ and adenocarcinoma $(O R=2.25)$. Otherwise, positive associations between history of breast $(O R=1.99)$ and total $(O R=1.71)$ cancers in siblings and the risk of male adenocarcinoma were observed. These results suggest that inherited genetic susceptibility may contribute to the development of lung cancer. In men, shared exposure to environmental factors among siblings may also be responsible for the increase in lung cancer risk.

Keywords: adenocarcinoma; case-control study; family history; lung cancer; smoking

Tohoku J. Exp. Med., 2019 February, 247 (2), 99-110. (C) 2019 Tohoku University Medical Press

\section{Introduction}

Lung cancer is a major site in cancer incidence worldwide (Curado et al. 2007). In Japan, it has the second highest incidence after stomach cancer in men and is the fifth leading-site in women (Hori et al. 2015).

Cigarette smoking has been regarded as the most important risk factor for lung cancer (International Agency for Research on Cancer 2004), and therefore smoking control is considered to be the effective method of prevention. In fact, the incidence of lung cancer seems to have pla- teaued recently in developed countries, possibly due to the decrease of smokers (Katanoda et al. 2013). However, the patterns of lung cancer incidence are not straightforward; the incidences of both squamous cell and small cell carcinoma have decreased, whereas that of adenocarcinoma has increased (Kinoshita et al. 2016). It is known that the association of smoking with lung cancer risk is weak for adenocarcinoma in comparison with other histologic types (Pesch et al. 2012; Seki et al. 2013). Furthermore, it has been indicated that the proportion of lung cancer patients who have never smoked varies from region to region (Parkin et al.

Received December 14, 2018; revised and accepted January 30, 2019. Published online February 19, 2019; doi: 10.1620/tjem.247.99.

Correspondence: Yuko Minami, Center for Preventive Medicine, Osaki Citizen Hospital, 2-3-15 Senjuji-machi, Furukawa, Osaki, Miyagi 989-6174, Japan.

e-mail: adym@med.tohoku.ac.jp 
2005; Subramanian and Govindan 2007; Wu et al. 2016; Lin et al. 2017). It has been estimated that $53 \%$ of all women with lung cancer worldwide are never smokers (Parkin et al. 2005; Subramanian and Govindan 2007), whereas the proportion in Japanese female patients is as high as 70\% (Sobue et al. 2002; Seki et al. 2013). Based on these observations, the distribution of other environmental factors including dust, chemical exposure and diet, rather than smoking, determine the patterns of lung cancer incidence (Hamra et al. 2014; Vieira et al. 2016). On the other hand, individuals may differ in their genetic susceptibility to environmental factors including tobacco smoke, and this may also have some impact on the incidence (Bartsch et al. 2000; Brennan et al. 2011).

In the assessment of genetic susceptibility, family history has been used as a surrogate for genetic risk (American Society of Clinical Oncology 2003; Khoury et al. 2005). In 1963, Tokuhata and Lilienfeld found evidence for familial aggregation of lung cancer (Tokuhata and Lilienfeld 1963), and since then several epidemiologic studies have evaluated lung cancer risk in relation to family history of lung cancer (Matakidou et al. 2005b; Nitadori et al. 2006; Coté et al. 2012). The associations between family histories of other cancers and lung cancer risk have also been investigated (Kunitoh et al. 1999; Poole et al. 1999). Most studies have reported an increase of overall lung cancer risk for individuals with family history of lung cancer (Matakidou et al. 2005b; Coté et al. 2012). Some studies have been stratified according to histology, type of relatives (father, mother, siblings, etc.), and smoking status (Nitadori et al. 2006; Coté et al. 2012). For example, a recent study conducted in Europe reported a strong association between family history of lung cancer and the risk of squamous cell carcinoma (Lissowska et al. 2010). A pooled analysis by the international consortium demonstrated a higher lung cancer risk for individuals with family history of lung cancer in siblings (Coté et al. 2012). However, there have been some differences in results among these previous studies (Nitadori et al. 2006; Lissowska et al. 2010; Coté et al. 2012). Few epidemiologic studies have investigated familial risk in Japanese population (Tsugane et al. 1987; Kunitoh et al. 1999; Nitadori et al. 2006). Furthermore, risk evaluation according to sex and histology has rarely been performed (Ambrosone et al. 1993). Existing evidence seems insufficient to conclude that family history has significant effects on lung cancer risk.

In the present hospital-based case-control study, we attempted to clarify the association between family history of cancer and lung cancer risk according to sex and histologic type. Data were obtained from patients aged 30 years and over who were admitted to a single hospital in Miyagi Prefecture, Japan, between 1997 and 2009. Lung cancer risk was evaluated in relation to family histories of lung and other major cancers.

\section{Methods}

\section{Data collection}

The data collection procedure we employed has been reported previously (Minami and Tateno 2003; Seki et al. 2013; Takizawa et al. 2018). Briefly, between January 1997 and December 2009, information on lifestyle, personal history and family history of diseases including cancer was collected from patients at their first admission to the Miyagi Cancer Center Hospital (MCCH) using a self-administered questionnaire. The questionnaire was distributed to 23,531 firstadmitted patients, of whom 21,056 responded.

\section{Study subjects}

Cases and controls were selected from among the respondents to the above questionnaire. To identify incident cases of lung cancer, a list of the respondents was linked to the hospital-based cancer registry files. Through this linkage, 1,733 patients aged 30 years and over (1,194 men, 539 women) were identified as cases. Among these cases, $1621(93.5 \%)$ were histopathologically confirmed: squamous cell carcinoma in 434, small cell carcinoma in 161, adenocarcinoma in 883 , large cell carcinoma in 44 , and others in 99 . For the remaining 112 cases $(6.5 \%)$, histopathologic data were unavailable.

Controls were selected from among the non-cancer respondents. Diagnoses of the non-cancer respondents were confirmed using the disease registration database at the $\mathrm{MCCH}$. Accordingly, 6,643 noncancer patients aged 30 years and over (3,483 men, 3,160 women) were identified as possible controls. The diagnoses among the possible controls were benign tumor in 3,638 (54.8\%), cardiovascular disease in $216(3.2 \%)$, respiratory tract disease in 280 (4.2\%), other benign disease in $1,778(26.8 \%)$, and no abnormal findings in 731 $(11.0 \%)$. The sites of benign tumors were the stomach in 308 , colorectum in 1,347 , lung in 36 , breast in 36 , gynecologic organs in 375 , bone or connective tissue in 985 , and other in 551 .

In each analysis for evaluating the risk related to family history, patients with benign tumors in the index site were excluded from possible controls because the risk of benign tumor may be related to family history of cancer (Minami et al. 1998). It is likely that the validity of the study would have been improved by such exclusion (Rothman and Greenland 1998).

This study was approved by the ethical review board of the Miyagi Cancer Center (Protocol Identification Number 23-50, January 19, 2012). The purpose of the survey was explained on the cover page of the questionnaire. We considered the return of questionnaires signed by the patients to imply their consent to participate in the study. The final response rate for the questionnaire survey was $84.7 \%$ for the cases and $89.8 \%$ for the possible controls.

Assessment of family history and statistical analysis

Information on family history of cancer in first-degree relatives (father, mother, and siblings) was obtained from the questionnaire survey. A positive answer to family history-related items was regarded as a positive history. Information on cancer sites in affected relatives was also obtained.

The present study evaluated lung cancer risk in relation to family history of cancer at following sites: lung, stomach, breast, and all sites. Stomach cancer is the leading site in male cancer incidence in Japan, whereas breast cancer incidence is highest in women. Using unconditional logistic regression analysis (Breslow and Day 1980), we estimated overall lung cancer risk and histology-specific risk 
(squamous cell carcinoma, small cell carcinoma, and adenocarcinoma) among men and women. To investigate whether ORs for family history (present vs. absent) were constant across both sexes, statistical significance was tested for the interaction term (sex*family history of cancer). Furthermore, to investigate synergistic effect between smoking status and family history of lung cancer, the analysis stratified by smoking status (ever, never) was performed. Stratification by passive smoking from spouses was performed for non-smoking married subjects.

The analyses were conducted according to the type of relatives, i.e., history in siblings only and in the father or mother (parental history), along with overall history in first-degree relatives. The following variables were considered to be confounders: age, year of recruitment, area of residence, referral status, occupation, alcohol drinking, pack-years of smoking, passive smoking from spouses, and birthplace. In evaluating the risk for family history in siblings only, subjects not having siblings were excluded and the number of siblings was added as a confounder. Missing values were treated as an additional variable category.

All analyses were performed using SAS version 9.3 (SAS Institute, Cary, NC). Values were regarded as significant if the twosided $P$ values were $<0.05$.

\section{Results}

The characteristics of the study subjects are presented in Table 1. The table shows the data for controls including patients with benign tumor (possible controls). The cases with squamous cell or small cell carcinoma tended to be smokers. On the other hand, the distribution of smokers in cases with adenocarcinoma was comparable to the controls.

Table 2 shows overall lung cancer risk in relation to family history of cancer at any site among men and women. In both men and women, family history of lung cancer in first-degree relatives was associated with an increased risk of lung cancer (men, OR $=1.43, P<0.05$; women, OR $=1.40, P=0.06$ ). According to the type of relatives, history of lung cancer in siblings only was significantly associated with male lung cancer risk. A significant positive association for parental history was found in women. There was no association between family history of stomach cancer and lung cancer risk in both sexes. Family history of breast cancer in first-degree relatives was associated with an increased risk of male lung cancer. This association was evident for a history in siblings only. The interaction term suggested that the effect of family history of breast cancer on overall lung cancer risk might significantly differ between men and women $(P$ for interaction $=0.04)$. A significant positive association between family history of cancers in all sites (total cancers) and lung cancer risk was also observed in males only.

Table 3 shows lung cancer risk according to histologic type. For adenocarcinoma, a positive association with family history of lung cancer in first-degree relatives was observed in both men $(P<0.05)$ and women $(P=0.08)$. According to the type of relatives, a higher risk for history in siblings only was found in men, whereas a significant positive association with parental history was observed in women. For squamous cell carcinoma, a significant positive association with parental history was observed in women; however, the confidence interval was wide due to the small number of cases $(n=3)$. For small cell carcinoma, an elevated risk for history in siblings only was observed in men.

Table 4 shows histology-specific lung cancer risk in relation to family histories of other cancers. Regarding family history of breast cancer, a significant positive association with history in first-degree relatives was found for male adenocarcinoma. This association was evident for history in siblings only. A positive association with history of breast cancer in siblings only was also observed for male small cell carcinoma. Conversely, such positive associations with family history of breast cancer were not observed for any histologic type of female lung cancer. The interaction term suggested that the effect of history of breast cancer in siblings only on the risk of adenocarcinoma might significantly differ between men and women ( $P$ for interaction $=0.04)$. Family history of total cancers was significantly associated with an increased risk of male adenocarcinoma. According to the type of relatives, a higher risk of adenocarcinoma was found for history in siblings only (OR $=1.71,95 \%$ CI: $1.25-2.33$ ).

Table 5 shows histology-specific lung cancer risk stratified by smoking status in relation to family history of lung cancer. Although the risks of squamous cell and small cell carcinoma could not be fully investigated due to the small number of cases, some differences in histology-specific risk were found between never- and ever-smokers. For female adenocarcinoma, a significant positive association for parental history of lung cancer was found among eversmokers $(\mathrm{OR}=4.13,95 \% \mathrm{CI}$ : $1.58-10.79)$. No such association was observed among never-smokers, suggesting synergistic interaction between smoking status and parental history of lung cancer $(P$ for interaction $=0.08$ ). On the other hand, stratified analysis in men showed that parental history was associated with an increased risk of adenocarcinoma among never-smokers, being inconsistent with the above-mentioned result in women.

The risk stratified by passive smoking status is shown in Table 6. The table shows only the results for the risk of adenocarcinoma. In non-smoking married women exposed to their husbands' tobacco smoke, a non-significant positive association was observed between parental history of lung cancer and the risk of adenocarcinoma $(\mathrm{OR}=1.80)$.

\section{Discussion}

The present study clarified lung cancer risk associated with family history of lung cancer according to sex and histologic type. The associations of family history of other cancers, including stomach, breast and total cancers, with lung cancer risk were also clarified. These data provide additional evidence for familial and genetic risk of lung cancer.

Regarding lung cancer risk in relation to family history 
Table 1. Characteristics of cases and controls by sex and histologic type.

\begin{tabular}{|c|c|c|c|c|c|c|c|c|c|c|}
\hline \multirow{4}{*}{ Factor } & \multicolumn{8}{|c|}{ Cases } & \multirow{3}{*}{\multicolumn{2}{|c|}{ Controls $^{\mathrm{b}}$}} \\
\hline & \multirow{2}{*}{\multicolumn{2}{|c|}{$\mathrm{All}^{\mathrm{a}}$}} & \multicolumn{6}{|c|}{ Histologic type } & & \\
\hline & & & \multicolumn{2}{|c|}{ Squamous cell } & \multicolumn{2}{|c|}{ Small cell } & \multicolumn{2}{|c|}{ Adeno } & & \\
\hline & $\mathrm{M}^{\mathrm{c}}$ & $\mathrm{W}^{\mathrm{d}}$ & $\mathrm{M}$ & $\mathrm{W}$ & $\mathrm{M}$ & $\mathrm{W}$ & $\mathrm{M}$ & $\mathrm{W}$ & $\mathrm{M}$ & $\mathrm{W}$ \\
\hline Number of subjects & 1194 & 539 & 391 & 43 & 134 & 27 & 491 & 392 & 3483 & 3160 \\
\hline \multicolumn{11}{|l|}{ Age group (years old) (\%) } \\
\hline $30-39$ & 0.6 & 0.7 & 0.5 & 0.0 & 0.0 & 0.0 & 0.8 & 1.0 & 5.8 & 8.5 \\
\hline $40-49$ & 3.4 & 5.4 & 1.8 & 0.0 & 3.7 & 7.4 & 3.7 & 5.4 & 11.4 & 17.6 \\
\hline $50-59$ & 15.6 & 17.3 & 8.7 & 11.6 & 22.4 & 11.1 & 19.4 & 18.1 & 22.4 & 22.1 \\
\hline $60-69$ & 28.2 & 29.1 & 24.5 & 23.3 & 38.1 & 25.9 & 28.9 & 32.6 & 30.1 & 25.3 \\
\hline $70-79$ & 43.1 & 38.6 & 53.2 & 46.5 & 29.1 & 48.2 & 40.7 & 36.0 & 24.4 & 20.3 \\
\hline $80-$ & 9.1 & 8.9 & 11.3 & 18.6 & 6.7 & 7.4 & 6.5 & 6.9 & 5.9 & 6.2 \\
\hline Mean (years old) & 68.3 & 67.2 & 70.7 & 71.0 & 66.1 & 67.9 & 67.0 & 66.4 & 61.8 & 59.5 \\
\hline SD & 9.8 & 10.5 & 8.4 & 9.5 & 9.6 & 10.0 & 9.9 & 10.2 & 12.6 & 13.7 \\
\hline \multicolumn{11}{|l|}{ Year of recruitment (\%) } \\
\hline 1997-2002 & 34.4 & 36.6 & 32.2 & 32.6 & 43.3 & 44.4 & 35.2 & 37.0 & 51.8 & 54.7 \\
\hline 2003-2009 & 65.6 & 63.4 & 67.8 & 67.4 & 56.7 & 55.6 & 64.8 & 63.0 & 48.2 & 45.3 \\
\hline \multicolumn{11}{|l|}{ Area of residence (\%) } \\
\hline Southern Miyagi prefecture & 77.8 & 82.6 & 73.1 & 79.1 & 82.8 & 88.9 & 80.5 & 81.9 & 88.9 & 88.3 \\
\hline Other area & 22.2 & 17.4 & 26.9 & 20.9 & 17.2 & 11.1 & 19.5 & 18.1 & 11.1 & 11.7 \\
\hline \multicolumn{11}{|l|}{ Referral status (\%) } \\
\hline From screening & 18.3 & 31.9 & 17.1 & 11.6 & 11.2 & 14.8 & 22.2 & 35.7 & 17.7 & 18.1 \\
\hline Other & 81.7 & 68.1 & 82.9 & 88.4 & 88.8 & 85.2 & 77.8 & 64.3 & 82.3 & 81.9 \\
\hline \multicolumn{11}{|l|}{ Occupation (\%) } \\
\hline Professional or clerical work & 33.0 & 24.1 & 28.9 & 27.9 & 30.6 & 33.3 & 36.3 & 22.7 & 42.7 & 34.0 \\
\hline Industrial work or fishery & 35.2 & 15.8 & 30.7 & 2.3 & 33.6 & 7.4 & 38.1 & 17.9 & 34.2 & 14.1 \\
\hline Agriculture or forestry & 16.5 & 9.5 & 21.2 & 4.7 & 25.4 & 0.0 & 11.8 & 11.0 & 12.7 & 10.0 \\
\hline Other $^{\mathrm{e}}$ & 2.1 & 26.9 & 2.6 & 25.6 & 1.5 & 33.3 & 2.0 & 27.5 & 1.6 & 25.0 \\
\hline Missing & 13.2 & 23.7 & 16.6 & 39.5 & 8.9 & 26.0 & 11.8 & 20.9 & 8.8 & 16.9 \\
\hline \multicolumn{11}{|l|}{ Birthplace (\%) } \\
\hline Urban & 16.5 & 16.2 & 15.1 & 25.6 & 12.7 & 7.4 & 18.9 & 16.1 & 16.1 & 16.3 \\
\hline Rural or other & 80.2 & 79.0 & 81.3 & 67.4 & 85.1 & 81.5 & 78.0 & 80.1 & 81.9 & 79.8 \\
\hline Missing & 3.3 & 4.8 & 3.6 & 7.0 & 2.2 & 11.1 & 3.1 & 3.8 & 2.0 & 3.9 \\
\hline \multicolumn{11}{|l|}{ Number of siblings ${ }^{\mathrm{f}}(\%)$} \\
\hline & 0.2 & 0.2 & 0.3 & 0.0 & 0.0 & 0.0 & 0.2 & 0.3 & 0.1 & 0.1 \\
\hline $1<<5$ & 32.7 & 34.3 & 27.6 & 27.9 & 36.6 & 48.2 & 36.9 & 34.9 & 41.2 & 47.0 \\
\hline & 57.9 & 57.5 & 63.4 & 67.4 & 55.2 & 44.4 & 53.3 & 56.4 & 50.3 & 46.2 \\
\hline Missing & 9.2 & 8.0 & 8.7 & 4.7 & 8.2 & 7.4 & 9.6 & 8.4 & 8.4 & 6.7 \\
\hline Alcohol drinking (\%) & & & & & & & & & & \\
\hline Ever & 71.2 & 21.0 & 69.6 & 27.9 & 75.4 & 33.3 & 72.9 & 19.9 & 75.9 & 23.3 \\
\hline Never & 25.4 & 71.2 & 26.8 & 60.5 & 23.1 & 59.3 & 23.4 & 72.5 & 21.5 & 71.3 \\
\hline Missing & 3.4 & 7.8 & 3.6 & 11.6 & 1.5 & 7.4 & 3.7 & 7.6 & 2.6 & 5.4 \\
\hline Smoking (\%) & & & & & & & & & & \\
\hline Ever & 89.8 & 23.6 & 93.9 & 58.1 & 96.2 & 85.2 & 84.1 & 16.1 & 76.0 & 15.6 \\
\hline Never & 7.3 & 71.2 & 3.3 & 34.9 & 3.0 & 14.8 & 12.2 & 78.3 & 22.0 & 80.0 \\
\hline Missing & 2.9 & 5.2 & 2.8 & 7.0 & 0.8 & 0.0 & 3.7 & 5.6 & 2.0 & 4.4 \\
\hline Pack-years of smoking (\%) & & & & & & & & & & \\
\hline 0 (Never smoked) & 7.3 & 71.2 & 3.3 & 34.9 & 3.0 & 14.8 & 12.2 & 78.3 & 22.0 & 80.0 \\
\hline $0<<8.5$ & 1.4 & 2.2 & 0.8 & 2.3 & 0.8 & 0.0 & 2.0 & 2.3 & 3.0 & 4.6 \\
\hline $8.5 \leq<19$ & 3.4 & 4.3 & 0.8 & 7.0 & 3.0 & 14.8 & 5.3 & 3.1 & 8.7 & 4.6 \\
\hline $19 \leq$ & 68.3 & 11.3 & 73.4 & 34.9 & 74.6 & 63.0 & 62.7 & 5.9 & 53.5 & 4.5 \\
\hline Ever smoked (missing for pack-years) & 16.7 & 5.8 & 18.9 & 13.9 & 17.8 & 7.4 & 14.1 & 4.8 & 10.8 & 1.9 \\
\hline Missing for smoking status & 2.9 & 5.2 & 2.8 & 7.0 & 0.8 & 0.0 & 3.7 & 5.6 & 2.0 & 4.4 \\
\hline Passive smoking from spouse (\%) & & & & & & & & & & \\
\hline Never & 73.6 & 21.0 & 77.3 & 11.6 & 72.4 & 11.1 & 70.4 & 21.7 & 75.7 & 24.6 \\
\hline Ever & 9.1 & 50.6 & 6.1 & 46.5 & 9.0 & 59.3 & 10.8 & 53.3 & 9.5 & 51.1 \\
\hline Missing & 9.1 & 18.9 & 9.2 & 27.9 & 11.9 & 25.9 & 9.4 & 16.1 & 6.1 & 15.3 \\
\hline Unmarried or unknown marital status & 8.2 & 9.5 & 7.4 & 14.0 & 6.7 & 3.7 & 9.4 & 8.9 & 8.7 & 9.0 \\
\hline
\end{tabular}

anclude all lung cancer patients (histologically confirmed 1,621, unconfirmed 112).

bInclude patients with benign tumor.

cM, men.

dW, women.

eHousehold wife / Domestic help / Other.

fIncludes study subject and its siblings. Data according to sisiters and brothers were unavailable. 
Table 2. Odds ratio (OR) and $95 \%$ confidence interval (CI) according to family history of cancer by sex.

\begin{tabular}{|c|c|c|c|c|c|c|c|c|c|}
\hline & \multicolumn{4}{|c|}{ Men } & \multicolumn{4}{|c|}{ Women } & \multirow{2}{*}{ Interaction } \\
\hline & Cases & Controls $^{\mathrm{a}}$ & OR & $95 \% \mathrm{CI}$ & Cases & Controls & OR & $95 \% \mathrm{CI}$ & \\
\hline \multicolumn{10}{|l|}{$\begin{array}{l}\text { Cancer site in family history of cancer } \\
\text { Lung }\end{array}$} \\
\hline Absent & 1,070 & 3,224 & 1 & (reference) & 493 & 2,951 & 1 & (reference) & \\
\hline Present in first-degree relatives ${ }^{\mathrm{b}}$ & 124 & 243 & 1.43 & $1.12-1.83$ & 46 & 189 & 1.40 & $0.98-2.01$ & 0.98 \\
\hline in siblings only ${ }^{\mathrm{c}}$ & 71 & 91 & 1.81 & $1.28-2.56$ & 19 & 69 & 1.16 & $0.68-2.00$ & 0.22 \\
\hline in father or mother ${ }^{b}$ & 53 & 152 & 1.17 & $0.83-1.65$ & 27 & 120 & 1.62 & $1.02-2.57$ & 0.22 \\
\hline \multicolumn{10}{|l|}{ Stomach } \\
\hline Absent & 1,004 & 2,837 & 1 & (reference) & 454 & 2,585 & 1 & (reference) & \\
\hline Present in first-degree relatives & 190 & 484 & 1.04 & $0.85-1.26$ & 85 & 429 & 0.98 & $0.75-1.28$ & 0.97 \\
\hline in siblings only & 46 & 137 & 0.73 & $0.51-1.04$ & 24 & 125 & 0.78 & $0.49-1.25$ & 0.78 \\
\hline in father or mother & 144 & 347 & 1.19 & $0.95-1.49$ & 61 & 304 & 1.08 & $0.79-1.47$ & 0.88 \\
\hline \multicolumn{10}{|l|}{ Breast } \\
\hline Absent & 1,158 & 3,407 & 1 & (reference) & 520 & 3,001 & 1 & (reference) & \\
\hline Present in first-degree relatives & 36 & 76 & 1.83 & $1.05-3.19$ & 19 & 123 & 0.78 & $0.43-1.42$ & 0.04 \\
\hline in siblings only & 26 & 45 & 1.99 & $1.02-3.90$ & 13 & 83 & 0.70 & $0.34-1.43$ & 0.04 \\
\hline in father or mother & 10 & 31 & 1.72 & $0.65-4.57$ & 6 & 40 & 1.02 & $0.35-3.00$ & 0.47 \\
\hline \multicolumn{10}{|l|}{ All sites } \\
\hline Absent & 640 & 1,010 & 1 & (reference) & 286 & 783 & 1 & (reference) & \\
\hline Present in first-degree relatives & 554 & 659 & 1.24 & $1.05-1.46$ & 253 & 578 & 1.02 & $0.82-1.27$ & 0.37 \\
\hline in siblings only & 197 & 195 & 1.31 & $1.02-1.67$ & 89 & 168 & 1.08 & $0.78-1.49$ & 0.72 \\
\hline in father or mother & 357 & 464 & 1.18 & $0.98-1.43$ & 164 & 410 & 0.99 & $0.77-1.27$ & 0.40 \\
\hline
\end{tabular}

an statistical analysis for each site, subjects with benign tumor in the site were excluded from controls.

bOR was adjusted for age, year of recruitment, area of residence (southern Miyagi Prefecture, other area), referral status (from screening, other), occupation (professional or clerical work, industrial work or fishery, agriculture or forestry, other), birthplace (urban, rural or other), alcohol drinking (never, ever), pack-years of smoking $(0,0<<8.5,8.5 \leq<19,19 \leq)$, and passive smoking from spouse (ever, never, unmarried or unknown marital status).

'Subjects not having siblings were excluded. OR was adjusted for age, year of recruitment, area of residence (southern Miyagi Prefecture, other area), referral status (from screening, other), occupation (professional or clerical work, industrial work or fishery, agriculture or forestry, other), birthplace (urban, rural or other), number of siblings $(1<<5,5 \leq$ ), alcohol drinking (never, ever), pack-years of smoking $(0,0<<8.5,8.5 \leq<19,19 \leq)$, and passive smoking from spouse (ever, never, unmarried or unknown marital status).

dP for interaction between sex and family history.

Table 3. Odds ratio (OR) and 95\% confidence interval (CI) according to family history of lung cancer by sex and histologic type.

\begin{tabular}{|c|c|c|c|c|c|c|c|c|c|c|}
\hline & \multirow{2}{*}{ Controls $^{\mathrm{a}}$} & \multicolumn{3}{|c|}{ Squamous cell } & \multicolumn{3}{|c|}{ Small cell } & \multicolumn{3}{|c|}{ Adeno } \\
\hline & & Cases & OR & $95 \% \mathrm{CI}$ & Cases & OR & $95 \% \mathrm{CI}$ & Cases & OR & $95 \% \mathrm{CI}$ \\
\hline \multicolumn{11}{|l|}{ Men } \\
\hline Absent & 3,224 & 350 & 1 & (reference) & 122 & 1 & (reference) & 432 & 1 & (reference) \\
\hline Present in first-degree relatives ${ }^{\mathrm{b}}$ & 243 & 41 & 1.37 & $0.93-2.01$ & 12 & 1.28 & $0.69-2.38$ & 59 & 1.69 & $1.24-2.32$ \\
\hline in siblings only ${ }^{\mathrm{c}}$ & 91 & 23 & 1.38 & $0.82-2.32$ & 9 & 2.28 & $1.09-4.76$ & 33 & 2.25 & $1.45-3.48$ \\
\hline in father or mother ${ }^{b}$ & 152 & 18 & 1.38 & $0.80-2.38$ & 3 & 0.55 & $0.17-1.78$ & 26 & 1.37 & $0.88-2.14$ \\
\hline \multicolumn{11}{|l|}{ Women } \\
\hline Absent & 2,951 & 39 & 1 & (reference) & 25 & 1 & (reference) & 359 & 1 & (reference) \\
\hline Present in first-degree relatives & 189 & 4 & 2.10 & $0.69-6.36$ & 2 & 0.78 & $0.15-4.05$ & 33 & 1.44 & $0.96-2.16$ \\
\hline in siblings only & 69 & 1 & 0.73 & $0.09-5.96$ & 0 & - & $-\quad-$ & 13 & 1.14 & $0.61-2.13$ \\
\hline in father or mother & 120 & 3 & 3.87 & $1.05-14.30$ & 2 & 1.23 & $0.22-6.99$ & 20 & 1.72 & $1.03-2.88$ \\
\hline \multicolumn{11}{|c|}{$P$ for interaction between sex and family history } \\
\hline in first-degree relatives & & & 0.47 & & & 0.99 & & & 0.47 & \\
\hline in siblings only & & & 0.60 & & & - & & & 0.12 & \\
\hline in father or mother & & & 0.14 & & & 0.19 & & & 0.58 & \\
\hline
\end{tabular}

aSubjects with lung benign tumor were excluded from controls.

${ }^{b} \mathrm{OR}$ was adjusted for age, year of recruitment, area of residence (southern Miyagi Prefecture, other area), referral status (from screening, other), occupation (professional or clerical work, industrial work or fishery, agriculture or forestry, other), birthplace (urban, rural or other), alcohol drinking (never, ever), pack-years of smoking $(0,0<<8.5,8.5 \leq<19,19 \leq)$, and passive smoking from spouse (ever, never, unmarried or unknown marital status).

'Subjects not having siblings were excluded. OR was adjusted for age, year of recruitment, area of residence (southern Miyagi Prefecture, other area), referral status (from screening, other), occupation (professional or clerical work, industrial work or fishery, agriculture or forestry, other), birthplace (urban, rural or other), number of siblings $(1<<5,5 \leq$ ), alcohol drinking (never, ever), pack-years of smoking $(0,0<<8.5,8.5 \leq<19,19 \leq)$, and passive smoking from spouse (ever, never, unmarried or unknown marital status). 
Table 4. Odds ratio (OR) and 95\% confidence interval (CI) according to family history of cancer of stomach and breast, and cancer in all sites by sex and histologic type.

\begin{tabular}{|c|c|c|c|c|c|c|c|c|c|c|}
\hline & \multirow{2}{*}{ Controls $^{\mathrm{a}}$} & \multicolumn{3}{|c|}{ Squamous cell } & \multicolumn{3}{|c|}{ Small cell } & \multicolumn{3}{|c|}{ Adeno } \\
\hline & & Cases & OR & $95 \% \mathrm{CI}$ & Cases & OR & $95 \% \mathrm{CI}$ & Cases & OR & $95 \% \mathrm{CI}$ \\
\hline \multicolumn{11}{|l|}{ Family history of stomach cancer } \\
\hline \multicolumn{11}{|l|}{ Men } \\
\hline Absent & 2,837 & 326 & 1 & (reference) & 112 & 1 & (reference) & 416 & 1 & (reference) \\
\hline Present in first-degree relatives ${ }^{b}$ & 484 & 65 & 1.07 & $0.78-1.45$ & 22 & 1.11 & $0.69-1.79$ & 75 & 1.01 & $0.77-1.32$ \\
\hline in siblings only ${ }^{\mathrm{c}}$ & 137 & 15 & 0.70 & $0.39-1.24$ & 5 & 0.73 & $0.29-1.85$ & 20 & 0.85 & $0.52-1.40$ \\
\hline in father or mother ${ }^{b}$ & 347 & 50 & 1.27 & $0.89-1.79$ & 17 & 1.28 & $0.75-2.18$ & 55 & 1.08 & $0.79-1.48$ \\
\hline \multicolumn{11}{|l|}{ Women } \\
\hline Absent & 2,585 & 40 & 1 & (reference) & 24 & 1 & (reference) & 322 & 1 & (reference) \\
\hline Present in first-degree relatives & 429 & 3 & 0.46 & $0.14-1.54$ & 3 & 0.86 & $0.23-3.19$ & 70 & 1.11 & $0.83-1.48$ \\
\hline in siblings only & 125 & 2 & 0.77 & $0.17-3.45$ & 1 & 0.70 & $0.07-6.79$ & 19 & 0.84 & $0.50-1.41$ \\
\hline in father or mother & 304 & 1 & 0.24 & $0.03-1.81$ & 2 & 0.96 & $0.20-4.62$ & 51 & 1.25 & $0.89-1.74$ \\
\hline \multicolumn{11}{|c|}{$P$ for interaction between sex and family history } \\
\hline in first-degree relatives & & & 0.16 & & & 0.59 & & & 0.48 & \\
\hline in siblings only & & & 0.92 & & & 0.98 & & & 0.85 & \\
\hline in father or mother & & & 0.12 & & & 0.54 & & & 0.44 & \\
\hline \multicolumn{11}{|l|}{ Family history of breast cancer } \\
\hline \multicolumn{11}{|l|}{ Men } \\
\hline Absent & 3,407 & 383 & 1 & (reference) & 129 & 1 & (reference) & 474 & 1 & (reference) \\
\hline Present in first-degree relatives & 76 & 8 & 1.05 & $0.48-2.32$ & 5 & 1.88 & $0.73-4.87$ & 17 & 1.83 & $1.05-3.19$ \\
\hline in siblings only & 45 & 5 & 0.87 & $0.32-2.32$ & 5 & 2.78 & $1.04-7.44$ & 12 & 1.99 & $1.02-3.90$ \\
\hline in father or mother & 31 & 3 & 1.48 & $0.39-5.60$ & 0 & - & - & 5 & 1.72 & $0.65-4.57$ \\
\hline \multicolumn{11}{|l|}{ Women } \\
\hline Absent & 3,001 & 41 & 1 & (reference) & 25 & 1 & (reference) & 379 & 1 & (reference) \\
\hline Present in first-degree relatives & 123 & 2 & 0.91 & $0.20-4.14$ & 2 & 1.31 & $0.25-6.90$ & 13 & 0.78 & $0.43-1.42$ \\
\hline in siblings only & 83 & 1 & 0.52 & $0.06-4.30$ & 1 & 0.72 & $0.08-6.92$ & 9 & 0.70 & $0.34-1.43$ \\
\hline in father or mother & 40 & 1 & 2.28 & $0.24-21.42$ & 1 & 5.78 & $0.50-67.16$ & 4 & 1.02 & $0.35-3.00$ \\
\hline \multicolumn{11}{|c|}{$P$ for interaction between sex and family history } \\
\hline in first-degree relatives & & & 0.99 & & & 0.68 & & & 0.04 & \\
\hline in siblings only & & & 0.80 & & & 0.30 & & & 0.04 & \\
\hline in father or mother & & & 0.73 & & & - & & & 0.47 & \\
\hline \multicolumn{11}{|l|}{ Family history of cancer in all sites } \\
\hline \multicolumn{11}{|l|}{ Men } \\
\hline Absent & 1,010 & 214 & 1 & (reference) & 74 & 1 & (reference) & 252 & 1 & (reference) \\
\hline Present in first-degree relatives & 659 & 177 & 1.11 & $0.87-1.43$ & 60 & 1.26 & $0.87-1.82$ & 239 & 1.33 & $1.07-1.65$ \\
\hline in siblings only & 195 & 58 & 0.94 & $0.65-1.37$ & 23 & 1.46 & $0.86-2.48$ & 95 & 1.71 & $1.25-2.33$ \\
\hline in father or mother & 464 & 119 & 1.19 & $0.90-1.58$ & 37 & 1.10 & $0.72-1.69$ & 144 & 1.18 & $0.92-1.52$ \\
\hline \multicolumn{11}{|l|}{ Women } \\
\hline Absent & 783 & 24 & 1 & (reference) & 13 & 1 & (reference) & 205 & 1 & (reference) \\
\hline Present in first-degree relatives & 578 & 19 & 0.99 & $0.49-1.98$ & 14 & 0.88 & $0.34-2.25$ & 187 & 1.08 & $0.85-1.38$ \\
\hline in siblings only & 168 & 8 & 1.08 & $0.41-2.82$ & 6 & 1.51 & $0.39-5.78$ & 60 & 1.06 & $0.73-1.52$ \\
\hline in father or mother & 410 & 11 & 0.85 & $0.37-1.98$ & 8 & 0.68 & $0.22-2.06$ & 127 & 1.08 & $0.82-1.41$ \\
\hline \multicolumn{11}{|c|}{$P$ for interaction between sex and family history } \\
\hline in first-degree relatives & & & 0.99 & & & 0.96 & & & 0.31 & \\
\hline in siblings only & & & 0.49 & & & 0.57 & & & 0.23 & \\
\hline in father or mother & & & 0.58 & & & 0.78 & & & 0.70 & \\
\hline
\end{tabular}

an statistical analysis for each site, subjects with benign tumor in the site were excluded from controls.

${ }^{\mathrm{b}} \mathrm{OR}$ was adjusted for age, year of recruitment, area of residence (southern Miyagi Prefecture, other area), referral status (from screening, other), occupation (professional or clerical work, industrial work or fishery, agriculture or forestry, other), birthplace (urban, rural or other), alcohol drinking (never, ever), pack-years of smoking $(0,0<<8.5,8.5 \leq<19,19 \leq$ ), and passive smoking from spouse (ever, never, unmarried or unknown marital status).

'Subjects not having siblings were excluded. OR was adjusted for age, year of recruitment, area of residence (southern Miyagi Prefecture, other area), referral status (from screening, other), occupation (professional or clerical work, industrial work or fishery, agriculture or forestry, other), birthplace (urban, rural or other), number of siblings $(1<<5,5 \leq$ ), alcohol drinking (never, ever), pack-years of smoking $(0,0<<8.5,8.5 \leq<19,19 \leq)$, and passive smoking from spouse (ever, never, unmarried or unknown marital status).

of lung cancer, the present study showed that family history of lung cancer in first-degree relatives was positively associated with the overall risk of lung cancer in both sexes. According to histologic type, the positive association tended to be evident for adenocarcinoma. Previous studies have consistently demonstrated an increased overall lung cancer risk among individuals with family history of lung cancer in first-degree relatives (Tokuhata and Lilienfeld 1963; Poole et al. 1999; Matakidou et al. 2005b; Nitadori et al. 2006; Lissowska et al. 2010; Coté et al. 2012). Meta- 
Table 5. Odds ratio (OR) and $95 \%$ confidence interval (CI) associated with family history of lung cancr according to smoking status by histologic type.

\begin{tabular}{|c|c|c|c|c|c|c|c|c|c|c|}
\hline & \multirow{2}{*}{ Controls ${ }^{i}$} & \multicolumn{3}{|c|}{ Squamous cell } & \multicolumn{3}{|c|}{ Small cell } & \multicolumn{3}{|c|}{ Adeno } \\
\hline & & Cases & OR & $95 \% \mathrm{CI}$ & Cases & OR & $95 \% \mathrm{CI}$ & Cases & OR & $95 \% \mathrm{CI}$ \\
\hline \multicolumn{11}{|l|}{$\overline{\text { Men }}$} \\
\hline \multicolumn{11}{|l|}{ Never-smokers } \\
\hline Absent & 718 & 12 & 1 & (reference) & 4 & 1 & (reference) & 51 & 1 & (reference) \\
\hline Present in first-degree relatives ${ }^{\mathrm{b}}$ & 47 & 1 & 1.32 & $0.15-11.40$ & 0 & - & $-\quad-$ & 9 & 3.31 & $1.44-7.60$ \\
\hline in siblings only ${ }^{\mathrm{c}}$ & 17 & 0 & - & - & 0 & - & - & 4 & 3.30 & $0.95-11.48$ \\
\hline in father or mother ${ }^{b}$ & 30 & 1 & 2.48 & $0.27-22.66$ & 0 & - & - & 5 & 3.60 & $1.23-10.50$ \\
\hline \multicolumn{11}{|l|}{ Ever-smokers } \\
\hline Absent & 2,446 & 329 & 1 & (reference) & 117 & 1 & (reference) & 364 & 1 & (reference) \\
\hline Present in first-degree relatives & 187 & 38 & 1.43 & $0.96-2.13$ & 12 & 1.40 & $0.75-2.61$ & 49 & 1.69 & $1.20-2.39$ \\
\hline in siblings only & 67 & 22 & 1.59 & $0.93-2.74$ & 9 & 2.64 & $1.25-5.60$ & 29 & 2.50 & $1.55-4.02$ \\
\hline in father or mother & 120 & 16 & 1.29 & $0.73-2.27$ & 3 & 0.59 & $0.18-1.90$ & 20 & 1.22 & $0.74-2.01$ \\
\hline \multicolumn{11}{|c|}{$P$ for interaction beween smoking and family history } \\
\hline in first degree relatives & & & 0.98 & & & 0.97 & & & 0.25 & \\
\hline in siblings only & & & 0.98 & & & 0.98 & & & 0.79 & \\
\hline in father or mother & & & 0.50 & & & 0.98 & & & 0.16 & \\
\hline \multicolumn{11}{|l|}{ Women } \\
\hline \multicolumn{11}{|l|}{ Never-smokers } \\
\hline Absent & 2,360 & 13 & 1 & (reference) & 4 & 1 & (reference) & 287 & 1 & (reference) \\
\hline Present in first-degree relatives & 153 & 2 & 2.52 & $0.55-11.61$ & 0 & - & - & 20 & 1.08 & $0.65-1.77$ \\
\hline in siblings only & 60 & 0 & - & - & 0 & - & - & 10 & 1.04 & $0.51-2.11$ \\
\hline in father or mother & 93 & 2 & 7.37 & $1.48-36.69$ & 0 & - & - & 10 & 1.14 & $0.57-2.25$ \\
\hline \multicolumn{11}{|l|}{ Ever-smokers } \\
\hline Absent & 454 & 24 & 1 & (reference) & 21 & 1 & (reference) & 52 & 1 & (reference) \\
\hline Present in first-degree relatives & 34 & 1 & 0.49 & $0.05-4.76$ & 2 & 0.70 & $0.12-4.00$ & 11 & 2.77 & $1.18-6.51$ \\
\hline in siblings only & 8 & 1 & 1.30 & $0.12-14.24$ & 0 & - & - & 2 & 0.92 & $0.16-5.28$ \\
\hline in father or mother & 26 & 0 & - & - & 2 & 1.22 & $0.19-7.78$ & 9 & 4.13 & $1.58-10.79$ \\
\hline \multicolumn{11}{|c|}{$P$ for interaction beween smoking and family history } \\
\hline in first degree relatives & & & 0.29 & & & - & & & 0.06 & \\
\hline in sibling only & & & - & & & - & & & 0.72 & \\
\hline in father or mother & & & - & & & - & & & 0.08 & \\
\hline
\end{tabular}

${ }^{a}$ Subjects with benign lung tumor were excluded from controls.

${ }^{b}$ OR was adjusted for age, year of recruitment, area of residence (southern Miyagi Prefecture, other area), referral status (from screening, other), occupation (professional or clerical work, industrial work or fishery, agriculture or forestry, other), birthplace (urban, rural or other), alcohol drinking (never, ever), and passive smoking from spouse (ever, never, unmarried or unknown marital status).

'Subjects not having siblings were excluded. OR was adjusted for age, year of recruitment, area of residence (southern Miyagi Prefecture, other area), referral status (from screening, other), occupation (professional or clerical work, industrial work or fishery, agriculture or forestry, other), birthplace (urban, rural or other), number of siblings $(1<<5,5 \leq$ ), alcohol drinking (never, ever), and passive smoking from spouse (ever, never, unmarried or unknown marital status).

analysis (Lissowska et al. 2010) and pooled analysis (Coté et al. 2012) including studies mainly from Western countries showed a relative risk ranging between 1.4 and 2.0 among men and women. Our estimate for overall lung cancer risk was similar to those in these studies. On the other hand, the risk according to histologic type has been inconsistent among previous studies. For example, in several case-only studies (Sellers et al. 1992; Kunitoh et al. 1999), subjects with small cell carcinoma tended to report a positive family history of lung cancer. Some studies including the Japanese cohort study (the JPHC study) (Shaw et al. 1991; Ambrosone et al. 1993; Matakidou et al. 2005b; Nitadori et al. 2006; Gao et al. 2009; Lissowska et al. 2010) have indicated that family history of lung cancer was associated with an increased risk of squamous cell carcinoma. Although the meta-analysis (Lissowska et al. 2010) and pooled analysis (Coté et al. 2012) indicated that family history of lung cancer was positively associated with lung cancer risk for all histologic types, the magnitude of risk varied among the individual studies included.

With regard to the sex difference in lung cancer risk, few studies have focused on the effect of family history. Especially, sex-specific lung cancer risk according to type of relatives has been unclear. The pooled analysis found an elevated risk of lung cancer among men with siblings affected by lung cancer (Coté et al. 2012), which was comparable to our finding, whereas the JPHC study produced a conflicting result: a significant positive association between history in siblings and female lung cancer risk (Nitadori et al. 2006). The present study observed some variations in lung cancer risk between men and women, although the interaction term (sex*family history) did not necessarily indicate clear sex difference. We interpreted our results in relation to family history of lung cancer, as follows. In men, family history of lung cancer in siblings only was positively associated with overall lung cancer risk and the risk of small cell carcinoma and adenocarcinoma, which may have resulted mainly from common environmental 
Table 6. Odds ratio (OR) and $95 \%$ confidence interval (CI) associated with family history of lung cancer according to passive smoking status $^{\mathrm{a}}$ among non-smoking married subjects.

\begin{tabular}{|c|c|c|c|c|}
\hline & \multicolumn{4}{|c|}{ Adeno } \\
\hline & Cases & Control $^{b}$ & OR & $95 \% \mathrm{CI}$ \\
\hline \multicolumn{5}{|l|}{$\overline{\text { Men }}$} \\
\hline \multicolumn{5}{|l|}{ Never exposed to passive smoking } \\
\hline Absent & 41 & 574 & 1 & (reference) \\
\hline Present in first-degree relatives ${ }^{c}$ & 6 & 40 & 2.35 & $0.89-6.25$ \\
\hline in siblings only ${ }^{\mathrm{d}}$ & 3 & 15 & 2.37 & $0.60-9.43$ \\
\hline in father or mother ${ }^{\mathrm{c}}$ & 3 & 25 & 2.47 & $0.66-9.25$ \\
\hline \multicolumn{5}{|l|}{ Ever exposed to passive smoking } \\
\hline Absent & 1 & 24 & 1 & (reference) \\
\hline Present in first-degree relatives & 1 & 0 & - & - \\
\hline in siblings only & 0 & 0 & - & - \\
\hline in father or mother & 1 & 0 & - & - \\
\hline \multicolumn{5}{|l|}{$\begin{array}{l}P \text { for interaction beween passive } \\
\text { smoking and family history }\end{array}$} \\
\hline in first degree relatives & & & - & \\
\hline in siblings only & & & - & \\
\hline in father or mother & & & - & \\
\hline \multicolumn{5}{|l|}{ Women } \\
\hline \multicolumn{5}{|l|}{ Never exposed to passive smoking } \\
\hline Absent & 72 & 652 & 1 & (reference) \\
\hline Present in first-degree relatives & 3 & 40 & 0.66 & $0.19-2.26$ \\
\hline in siblings only & 2 & 13 & 1.25 & $0.26-5.97$ \\
\hline in father or mother & 1 & 27 & 0.35 & $0.04-2.68$ \\
\hline \multicolumn{5}{|l|}{ Ever exposed to passive smoking } \\
\hline Absent & 148 & 1,208 & 1 & (reference) \\
\hline Present in first-degree relatives & 13 & 74 & 1.60 & $0.84-3.04$ \\
\hline in siblings only & 6 & 29 & 1.42 & $0.56-3.62$ \\
\hline in father or mother & 7 & 45 & 1.80 & $0.77-4.21$ \\
\hline \multicolumn{5}{|l|}{$P$ for interaction beween passive } \\
\hline $\begin{array}{l}\text { smoking and family history } \\
\text { in first degree relatives }\end{array}$ & & & & \\
\hline in first degree relatives & & & 0.27 & \\
\hline in sibling only & & & 0.95 & \\
\hline in father or mother & & & 0.16 & \\
\hline
\end{tabular}

Due to the small number of cases, ORs could not be evaluated for squamous cell and small cell carcinoma.

aPassive smoking from spouses.

bSubjects with benign lung tumor were excluded from controls.

${ }^{\mathrm{C}} \mathrm{OR}$ was adjusted for age, year of recruitment, area of residence (southern Miyagi Prefecture, other area), referral status (from screening, other), occupation (professional or clerical work, industrial work or fishery, agriculture or forestry, other), birthplace (urban, rural or other), and alcohol drinking (never, ever).

dSubjects not having siblings were excluded. OR was adjusted for age, year of recruitment, area of residence (southern Miyagi Prefecture, other area), referral status (from screening, other), occupation (professional or clerical work, industrial work or fishery, agriculture or forestry, other), birthplace (urban, rural or other), number of siblings $(1<<5,5 \leq)$, and alcohol drinking (never, ever).

exposure among siblings, although genetic susceptibility may also play some roles. A previous study based on the Swedish Family-Cancer Database also reported significant positive associations between history of lung cancer in siblings and the risk of small cell carcinoma and adenocarcinoma; however, sex-specific risk was not presented (Li and Hemminki 2004). During life time period, some residential exposures including environmental smoke and lifestyles such as diet and smoking habit might have been shared among siblings. Early and long-term exposure to these shared factors may be responsible for an increased risk of small cell and adeno-carcinoma in men. For example, the detailed analysis of our data showed that male control subjects with history of lung cancer in siblings only tended to be long-term smokers, whereas no such tendency was observed for subjects with parental history of lung cancer (data not shown). Furthermore, these characteristics in male control subjects were not observed in female controls. 
Thus, men with siblings affected by lung cancer are likely to acquire lifestyles related to lung cancer risk, such as smoking, in later life. On the other hand, in women, parental history of lung cancer was associated with an increased risk of overall lung cancer, and squamous cell and adenocarcinoma, although the result for squamous cell carcinoma may have been a chance finding because of the small number of cases with parental history. From the view point of biological aspect, it has been hypothesized that women are more susceptible to environmental carcinogens than men (Mollerup et al. 2006; Kiyohara and Ohno 2010; Alberg et al. 2013). For adenocarcinoma, the association with active smoking, i.e. the most important environmental risk factor, has been known to be weak in comparison with other histologic types, as was shown in our previous study (Seki et al. 2013). Parental history is regarded as a surrogate for inherited genetic susceptibility. Therefore, inherited factors determining susceptibility to environmental carcinogens including tobacco smoke, or a cancer-predetermining gene, may play important roles in the development of female lung cancer, especially adenocarcinoma (Mollerup et al. 1999, 2006; Bartsch et al. 2000; Uppstad et al. 2011).

The results stratified by smoking status, as shown in Table 5, may provide clues for clarifying the complex interrelationships between environmental and inherited genetic factors that determine lung cancer risk. Previous studies have also attempted stratification of smoking. The results, however, have been inconsistent (Tokuhata and Lilienfeld 1963; Wu et al. 1996, 2016; Brownson et al. 1997; Etzel et al. 2003; Matakidou et al. 2005a; Nitadori et al. 2006; Gao et al. 2009; Rachtan et al. 2009; Coté et al. 2012; Lin et al. 2017). Although some studies have indicated the positive association between family history of lung cancer and lung cancer risk among never-smokers (Nitadori et al. 2006; Wu et al. 2016; Lin et al. 2017), the pooled analysis showed that family history of lung cancer was associated with an increased risk of lung cancer in both ever- and never-smokers (Coté et al. 2012). In the present study, family history of lung cancer was positively associated with the risk of adenocarcinoma among female ever-smokers. A large OR was found for parental history. Case-control studies from the UK and Poland also showed that female smokers with family history of lung cancer were at higher risk of lung cancer than those without such family history; however, data on the types of relative and histology were not presented in these studies (Matakidou et al. 2005a; Rachtan et al. 2009). Tobacco smoking is known to have a significant effect on the risk of adenocarcinoma for both sexes, although the magnitude of the effect is small as mentioned above (Seki et al. 2013). However, tobacco exposure levels in women are generally lower than in men. Based on these observations, it is likely that inherited genes, such as cytochrome P 450-related genes involved in the detoxification of tobacco smoke, may modify the susceptibility to tobacco carcinogenesis in female smokers (Mollerup et al. 1999, 2006; Bartsch et al. 2000; Uppstad et al. 2011). The role of these inherited genes may also be supported by the analysis stratified by passive smoking status as shown in Table 6 . Although hypothetically, women with parental history of lung cancer may have insufficient ability to detoxify tobacco smoke. In order to prevent lung cancer, they should be informed about the importance of smoking cessation and the avoidance of environmental tobacco smoke.

The present analysis on family histories of other cancers provides some suggestion of familial risk of lung cancer. First, history of breast cancer in siblings only was positively associated with male overall risk and the risk of male small cell carcinoma and adenocarcinoma. No such associations were observed in women. As stated above in relation to family history of lung cancer, this finding also suggests that shared exposure to certain environmental and residential factors among siblings may play roles in the development of male small cell carcinoma and adenocarcinoma. With regard to breast cancer, it has been hypothesized that early exposure to factors such as childhood diet and physical activity might be related to breast cancer risk in adulthood (Okasha et al. 2003; Colditz et al. 2014). There are some similarities in risk factors between breast cancer and lung adenocarcinoma (Monninkhof et al. 2007; Leitzmann et al. 2009). It is likely that shared exposure to early environmental factors may impact on the risk of both breast cancer and male lung cancer. Lung cancer risk in relation to family history of breast cancer has been investigated in some previous studies (Wu et al. 1996; Brownson et al. 1997; Mayne et al. 1999). The study from the USA indicated a positive association between maternal history, the history in sisters and lung cancer risk (Mayne et al. 1999). In Japan, a case-only study showed that women with a family history of breast cancer in first-degree relatives are at higher risk of adenocarcinoma than men, which was inconsistent with our results (Tsuchiya et al. 2007). From the viewpoint of genetic and environmental risk, the association between family history of breast cancer and lung cancer risk is interesting and important, and further studies of this issue are required. Second, family history of total cancer in siblings only was positively associated with the risk of male lung cancer, especially adenocarcinoma. This finding also supports the importance of shared exposure to environmental and residential factors among siblings in the development of male lung cancer.

The present study had both strengths and limitations. In hospital-based case-control studies like this one, some methodological limitations are likely to influence the results. First, we considered comparability between the cases and the controls. We selected the controls from among patients who had been admitted to the same hospitals as the cases. However, the distribution of risk factors among the control subjects might have differed from that in the general population. Therefore, to improve comparability between cases and controls, we excluded patients with benign tumors at the index site believed to have been related to family history from among the controls (Rothman 
and Greenland 1998). Furthermore, statistical analyses were appropriately controlled for background characteristics, such as area of residence and referral pattern. Second, all family history was self-reported; therefore, this information was never validated. However, since the questionnaire has been fulfilled at the first admission before any definite diagnosis or treatment at the $\mathrm{MCCH}$, misclassification of family history would have been unlikely to affect disease classification, that is, non-differential. Therefore, information bias is unlikely to have distorted the present results. Third, data on lifestyle and smoking history among parents and siblings was unavailable. Environmental tobacco smoke during early life is an important risk factor for lung cancer. Clustering of smoking habits in families has also been suggested, and some previous studies have considered this issue (Jonsson et al. 2004; Lorenzo Bermejo and Hemminki 2005; Gao et al. 2009; Lin et al. 2015). To precisely identify the genetic component of family history, it may be necessary to control for any family history of smoking. Fourth, this study was performed at a single hospital in Miyagi Prefecture. To validate the present results, further studies in other regions will be required.

One of our study strengths was that a relatively large number of cases and controls were included. Consequently, we were able to evaluate sex- and histology-specific lung cancer risk. Another strength was that confounders such as occupation, pack-years of smoking, and birthplace were controlled for in the analysis. Birthplace may be a surrogate for general environment during childhood. Previous studies have not considered the effects of these confounders.

In conclusion, this case-control study clarified the association of family history of cancer with histology-specific lung cancer risk among Japanese men and women. In general, family history of cancer tended to be positively associated with the risk of adenocarcinoma among both sexes. According to the type of relatives, parental history of lung cancer was significantly associated with an increased risk of female adenocarcinoma, and this significant association was limited to ever-smokers, suggesting that some inherited genetic factors may play roles in the development of female adenocarcinoma. In men, family histories of lung, breast and total cancers among siblings only were associated with an increased risk of adenocarcinoma. Shared exposures to environmental factors among siblings, as well as genetic susceptibility, may be responsible for the increase of risk in men. Any strategy aimed at prevention of lung cancer will need to consider the role of family cancer history.

\section{Acknowledgments}

This work was supported by Grants-in-Aid from the Japan Society for the Promotion of Science (JSPS) for Scientific Research (B) (16H05240).

\section{Conflict of Interest}

The authors declare no conflict of interest.

\section{References}

Alberg, A.J., Wallace, K., Silvestri, G.A. \& Brock, M.V. (2013) Invited commentary: the etiology of lung cancer in men compared with women. Am. J. Epidemiol., 177, 613-616.

Ambrosone, C.B., Rao, U., Michalek, A.M., Cummings, K.M. \& Mettlin, C.J. (1993) Lung cancer histologic types and family history of cancer. Analysis of histologic subtypes of 872 patients with primary lung cancer. Cancer, 72, 1192-1198.

American Society of Clinical Oncology (2003) American Society of Clinical Oncology policy statement update: genetic testing for cancer susceptibility. J. Clin. Oncol., 21, 2397-2406.

Bartsch, H., Nair, U., Risch, A., Rojas, M., Wikman, H. \& Alexandrov, K. (2000) Genetic polymorphism of CYP genes, alone or in combination, as a risk modifier of tobacco-related cancers. Cancer Epidemiol. Biomarkers Prev., 9, 3-28.

Brennan, P., Hainaut, P. \& Boffetta, P. (2011) Genetics of lungcancer susceptibility. Lancet Oncol., 12, 399-408.

Breslow, N.E. \& Day, N.E. (1980) Unconditional Logistic Regression for Large Strata. In The Analysis of Case-Control Studies. Statistical Methods in Cancer research, vol. 1, IARC, Lyon, pp. 191-246.

Brownson, R.C., Alavanja, M.C., Caporaso, N., Berger, E. \& Chang, J.C. (1997) Family history of cancer and risk of lung cancer in lifetime non-smokers and long-term ex-smokers. Int. J. Epidemiol., 26, 256-263.

Colditz, G.A., Bohlke, K. \& Berkey, C.S. (2014) Breast cancer risk accumulation starts early: prevention must also. Breast Cancer Res. Treat., 145, 567-579.

Coté, M.L., Liu, M., Bonassi, S., Neri, M., Schwartz, A.G., Christiani, D.C., Spitz, M.R., Muscat, J.E., Rennert, G., Aben, K.K., Andrew, A.S., Bencko, V., Bickeboller, H., Boffetta, P., Brennan, P., et al. (2012) Increased risk of lung cancer in individuals with a family history of the disease: a pooled analysis from the International Lung Cancer Consortium. Eur. J. Cancer, 48, 1957-1968.

Curado, M.P., Edwards, B., Shin, H.R., Storm, H., Ferlay, J., Heanue, M. \& Boyle, P. (2007) Cancer Incidence in Five Continents, vol. IX. IARC Scientific Publications No. 160, IARC, Lyon.

Etzel, C.J., Amos, C.I. \& Spitz, M.R. (2003) Risk for smokingrelated cancer among relatives of lung cancer patients. Cancer Res., 63, 8531-8535.

Gao, Y., Goldstein, A.M., Consonni, D., Pesatori, A.C., Wacholder, S., Tucker, M.A., Caporaso, N.E., Goldin, L. \& Landi, M.T. (2009) Family history of cancer and nonmalignant lung diseases as risk factors for lung cancer. Int. J. Cancer, 125, 146-152.

Hamra, G.B., Guha, N., Cohen, A., Laden, F., Raaschou-Nielsen, O., Samet, J.M., Vineis, P., Forastiere, F., Saldiva, P., Yorifuji, T. \& Loomis, D. (2014) Outdoor particulate matter exposure and lung cancer: a systematic review and meta-analysis. Environ. Health Perspect., 122, 906-911.

Hori, M., Matsuda, T., Shibata, A., Katanoda, K., Sobue, T. \& Nishimoto, H.; Japan Cancer Surveillance Research Group (2015) Cancer incidence and incidence rates in Japan in 2009: a study of 32 population-based cancer registries for the Monitoring of Cancer Incidence in Japan (MCIJ) project. Jpn. J. Clin. Oncol., 45, 884-891.

International Agency for Research on Cancer (2004) Tobacco Smoke and Involuntary Smoking. IARC Monographs on the Evaluation of Carcinogenic Risks to Humans, Volume 83, IARC, Lyon.

Jonsson, S., Thorsteinsdottir, U., Gudbjartsson, D.F., Jonsson, 
H.H., Kristjansson, K., Arnason, S., Gudnason, V., Isaksson, H.J., Hallgrimsson, J., Gulcher, J.R., Amundadottir, L.T., Kong, A. \& Stefansson, K. (2004) Familial risk of lung carcinoma in the Icelandic population. JAMA, 292, 2977-2983.

Katanoda, K., Matsuda, T., Matsuda, A., Shibata, A., Nishino, Y., Fujita, M., Soda, M., Ioka, A., Sobue, T. \& Nishimoto, H. (2013) An updated report of the trends in cancer incidence and mortality in Japan. Jpn. J. Clin. Oncol., 43, 492-507.

Khoury, M.J., Davis, R., Gwinn, M., Lindegren, M.L. \& Yoon, P. (2005) Do we need genomic research for the prevention of common diseases with environmental causes? Am. J. Epidemiol., 161, 799-805.

Kinoshita, F.L., Ito, Y. \& Nakayama, T. (2016) Trends in lung cancer incidence rates by histological type in 1975-2008: a population-based study in Osaka, Japan. J. Epidemiol., 26, 579-586.

Kiyohara, C. \& Ohno, Y. (2010) Sex differences in lung cancer susceptibility: a review. Gend. Med., 7, 381-401.

Kunitoh, H., Sekine, I., Kubota, K., Tamura, T., Shinkai, T., Kodama, T., Saijo, N., Naruke, T. \& Yamaguchi, N. (1999) Histologic types of lung carcinoma and related family history of anatomic sites and histologic types of cancers. Cancer, 86, $1182-1188$.

Leitzmann, M.F., Koebnick, C., Abnet, C.C., Freedman, N.D., Park, Y., Hollenbeck, A., Ballard-Barbash, R. \& Schatzkin, A. (2009) Prospective study of physical activity and lung cancer by histologic type in current, former, and never smokers. Am. J. Epidemiol., 169, 542-553.

Li, X. \& Hemminki, K. (2004) Inherited predisposition to early onset lung cancer according to histological type. Int. J. Cancer, 112, 451-457.

Lin, H., Huang, Y.S., Yan, H.H., Yang, X.N., Zhong, W.Z., Ye, H.W., Yang, J.J., Zhou, Q. \& Wu, Y.L. (2015) A family history of cancer and lung cancer risk in never-smokers: A clinicbased case-control study. Lung Cancer, 89, 94-98.

Lin, K.F., Wu, H.F., Huang, W.C., Tang, P.L., Wu, M.T. \& Wu, F.Z. (2017) Propensity score analysis of lung cancer risk in a population with high prevalence of non-smoking related lung cancer. BMC Pulm. Med., 17, 120.

Lissowska, J., Foretova, L., Dabek, J., Zaridze, D., SzeszeniaDabrowska, N., Rudnai, P., Fabianova, E., Cassidy, A., Mates, D., Bencko, V., Janout, V., Hung, R.J., Brennan, P. \& Boffetta, P. (2010) Family history and lung cancer risk: international multicentre case-control study in Eastern and Central Europe and meta-analyses. Cancer Causes Control, 21, 1091-1104.

Lorenzo Bermejo, J. \& Hemminki, K. (2005) Familial lung cancer and aggregation of smoking habits: a simulation of the effect of shared environmental factors on the familial risk of cancer. Cancer Epidemiol. Biomarkers Prev., 14, 1738-1740.

Matakidou, A., Eisen, T., Bridle, H., O’Brien, M., Mutch, R. \& Houlston, R.S. (2005a) Case-control study of familial lung cancer risks in UK women. Int. J. Cancer, 116, 445-450.

Matakidou, A., Eisen, T. \& Houlston, R.S. (2005b) Systematic review of the relationship between family history and lung cancer risk. Br. J. Cancer, 93, 825-833.

Mayne, S.T., Buenconsejo, J. \& Janerich, D.T. (1999) Familial cancer history and lung cancer risk in United States nonsmoking men and women. Cancer Epidemiol. Biomarkers Prev., 8, 1065-1069.

Minami, Y., Ohuchi, N., Taeda, Y., Fukao, A. \& Hisamichi, S. (1998) Risk factors for benign breast disease according to histopathological type: comparisons with risk factors for breast cancer. Jpn. J. Cancer Res., 89, 116-123.

Minami, Y. \& Tateno, H. (2003) Associations between cigarette smoking and the risk of four leading cancers in Miyagi Prefecture, Japan: a multi-site case-control study. Cancer Sci., 94, 540-547.

Mollerup, S., Berge, G., Baera, R., Skaug, V., Hewer, A., Phillips, D.H., Stangeland, L. \& Haugen, A. (2006) Sex differences in risk of lung cancer: Expression of genes in the PAH bioactivation pathway in relation to smoking and bulky DNA adducts. Int. J. Cancer, 119, 741-744.

Mollerup, S., Ryberg, D., Hewer, A., Phillips, D.H. \& Haugen, A. (1999) Sex differences in lung CYP1A1 expression and DNA adduct levels among lung cancer patients. Cancer Res., 59, 3317-3320.

Monninkhof, E.M., Elias, S.G., Vlems, F.A., van der Tweel, I., Schuit, A.J., Voskuil, D.W. \& van Leeuwen, F.E.; TFPAC (2007) Physical activity and breast cancer: a systematic review. Epidemiology, 18, 137-157.

Nitadori, J., Inoue, M., Iwasaki, M., Otani, T., Sasazuki, S., Nagai, K. \& Tsugane, S. (2006) Association between lung cancer incidence and family history of lung cancer: data from a largescale population-based cohort study, the JPHC study. Chest, 130, 968-975.

Okasha, M., McCarron, P., Gunnell, D. \& Smith, G.D. (2003) Exposures in childhood, adolescence and early adulthood and breast cancer risk: a systematic review of the literature. Breast Cancer Res. Treat., 78, 223-276.

Parkin, D.M., Bray, F., Ferlay, J. \& Pisani, P. (2005) Global cancer statistics, 2002. CA Cancer J. Clin., 55, 74-108.

Pesch, B., Kendzia, B., Gustavsson, P., Jockel, K.H., Johnen, G., Pohlabeln, H., Olsson, A., Ahrens, W., Gross, I.M., Bruske, I., Wichmann, H.E., Merletti, F., Richiardi, L., Simonato, L., Fortes, C., et al. (2012) Cigarette smoking and lung cancer: relative risk estimates for the major histological types from a pooled analysis of case-control studies. Int. J. Cancer, 131, 1210-1219.

Poole, C.A., Byers, T., Calle, E.E., Bondy, J., Fain, P. \& Rodriguez, C. (1999) Influence of a family history of cancer within and across multiple sites on patterns of cancer mortality risk for women. Am. J. Epidemiol., 149, 454-462.

Rachtan, J., Sokolowski, A., Niepsuj, S., Zemla, B. \& Zwierko, M. (2009) Familial lung cancer risk among women in Poland. Lung Cancer, 65, 138-143.

Rothman, K.J. \& Greenland, S. (1998) Modern Epidemiology, 2nd ed., Lippincott-Raven, Philadelphia, pp. 96-103.

Seki, T., Nishino, Y., Tanji, F., Maemondo, M., Takahashi, S., Sato, I., Kawai, M. \& Minami, Y. (2013) Cigarette smoking and lung cancer risk according to histologic type in Japanese men and women. Cancer Sci., 104, 1515-1522.

Sellers, T.A., Elston, R.C., Atwood, L.D. \& Rothschild, H. (1992) Lung cancer histologic type and family history of cancer. Cancer, 69, 86-91.

Shaw, G.L., Falk, R.T., Pickle, L.W., Mason, T.J. \& Buffler, P.A. (1991) Lung cancer risk associated with cancer in relatives. $J$. Clin. Epidemiol., 44, 429-437.

Sobue, T., Yamamoto, S., Hara, M., Sasazuki, S., Sasaki, S. \& Tsugane, S.; JPHC Study Group. Japanese Public Health Center (2002) Cigarette smoking and subsequent risk of lung cancer by histologic type in middle-aged Japanese men and women: the JPHC study. Int. J. Cancer, 99, 245-251.

Subramanian, J. \& Govindan, R. (2007) Lung cancer in never smokers: a review. J. Clin. Oncol., 25, 561-570.

Takizawa, Y., Kawai, M., Kakugawa, Y., Nishino, Y., Ohuchi, N. \& Minami, Y. (2018) Alcohol consumption and breast cancer risk according to hormone receptor status in Japanese women: a case-control study. Tohoku J. Exp. Med., 244, 63-73.

Tokuhata, G.K. \& Lilienfeld, A.M. (1963) Familial aggregation of lung cancer in humans. J. Natl. Cancer Inst., 30, 289-312.

Tsuchiya, M., Iwasaki, M., Otani, T., Nitadori, J., Goto, K., Nishiwaki, Y., Uchitomi, Y. \& Tsugane, S. (2007) Breast cancer in first-degree relatives and risk of lung cancer: assessment of the existence of gene sex interactions. Jpn. J. Clin. Oncol., 37, 419-423.

Tsugane, S., Watanabe, S., Sugimura, H., Arimoto, H., Shimosato, Y. \& Suemasu, K. (1987) Smoking, occupation and family history in lung cancer patients under fifty years of age. Jpn.J. 
Clin. Oncol., 17, 309-317.

Uppstad, H., Osnes, G.H., Cole, K.J., Phillips, D.H., Haugen, A. \& Mollerup, S. (2011) Sex differences in susceptibility to PAHs is an intrinsic property of human lung adenocarcinoma cells. Lung Cancer, 71, 264-270.

Vieira, A.R., Abar, L., Vingeliene, S., Chan, D.S., Aune, D., Navarro-Rosenblatt, D., Stevens, C., Greenwood, D. \& Norat, T. (2016) Fruits, vegetables and lung cancer risk: a systematic review and meta-analysis. Ann. Oncol., 27, 81-96.

Wu, A.H., Fontham, E.T., Reynolds, P., Greenberg, R.S., Buffler, P.,
Liff, J., Boyd, P. \& Correa, P. (1996) Family history of cancer and risk of lung cancer among lifetime nonsmoking women in the United States. Am. J. Epidemiol., 143, 535-542.

Wu, F.Z., Huang, Y.L., Wu, C.C., Tang, E.K., Chen, C.S., Mar, G.Y., Yen, Y. \& Wu, M.T. (2016) Assessment of selection criteria for low-dose lung screening CT among Asian ethnic groups in Taiwan: from mass screening to specific risk-based screening for non-smoker lung cancer. Clin. Lung Cancer, 17, e45-e56. 